\title{
Investigation of the kinematics of seed movement in the centrifugal distributor of a pneumatic seeder
}

\author{
Azamat E. Bogus ${ }^{1, *}$ \\ ${ }^{1}$ Chair of «Processes and machines in agribusiness» of the faculty of mechanization of Kuban State \\ Agrarian University named after I.T. Trubilin, 350044, Krasnodar, Russia
}

\begin{abstract}
Theoretical studies of the seed movement in the centrifugal seed distributor of a pneumgivenatic grain seeder have been performed. There were obtained the equations describing the process of seed movement in a centrifugal seed distributor which allow determining the optimal parameters of the pneumatic system of the seeder. The relationship between the operating and design parameters of the centrifugal distributor is given.
\end{abstract}

\section{Relevance of the research}

The most promising ones are pneumatic grain seeders, which, due to a common hopper and a pneumatic metering and distribution system, can reduce labor costs by up to $30 \%$ and increase the productivity of the sowing unit by up to $25 \%$ [3, 5]. However, despite all the advantages, pneumatic grain seeders do not always provide a high-quality distribution of seed material over the feeding area [6,7]. Improving the design of pneumatic grain seeders is impossible without theoretical justification of the process of seed distribution in a centrifugal seed distributor, which requires the development of a mathematical model of seed movement that provides the possibility of substantiating the design and operating parameters of a pneumatic grain seeder. Improving the quality of seed distribution through seed pipelines by substantiating the design and technological parameters of the centrifugal distribution device of a pneumatic grain seeder based on modeling the movement of seeds is relevant.

\section{Equation of a particle motion in a centrifugal seed distributor}

The movement of a particle in a centrifugal seed distributor of a pneumatic grain seeder [4] is described by the Lagrange method in a fixed coordinate system by the expression system of equations:

\footnotetext{
*Corresponding author: azamat089@gmail.com
} 


$$
\left\{\begin{array}{l}
\bar{x}=x(t) \\
\bar{y}=y(t) \\
\bar{z}=z(t)
\end{array}\right.
$$

Consider the movement of a single grain $A$ in a centrifugal seed distributor, which rotates clockwise with angular velocity $\omega$, while falling down relative to the axis $z$ (figure 1).

For the mathematical description of the grain in the distribution of seeds, position it in Cartesian coordinates so that the axis direction $O X$ coincides with the direction of the velocity at the point $A$ at the entrance to the distributor, the axis $O Y$ and the axis $\mathrm{OZ}$ coaxial with the axis of camera pointing down.

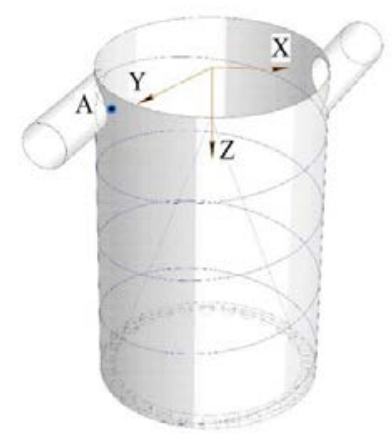

Fig. 1. Trajectory of the caryopsis movement in the centrifugal seed distributor

When considering the movement of the point $A$, we will take into account the initial moment of rotation at the outlet of the seed pipe. The movement of seeds from the opposite seed pipe will not be considered due to the fact that it will occur under the same conditions, only the initial coordinates will be different $X$ and $Y$.

The grain in the centrifugal distributor is affected by the following forces (Figure 2) [1]:

1) $F_{A}^{\tau}$ - tangential component of the driving aerodynamic force, $N$;

2) $F_{A}^{u \sigma}$ - centrifugal component of the driving aerodynamic force, $\mathrm{N}$;

3) $F_{a}^{c}$ - drag force, $\mathrm{N}$, directed in the opposite direction from the driving force, arising from the relative speed of the particle $A$ and air;

4) $F_{A}^{u}$ - Archimedes' centrifugal force, $\mathrm{H}$, directed along the radius to the center of rotation, which causes a change in the pressure of the carrier medium on the surface of the particle $A$;

5) $F_{g}$ - gravity force, $\mathrm{N}$, acting vertically downwards;

6) $F_{a p x}$ - Archimedes ' lifting force, $\mathrm{H}$, acting in the opposite direction of gravity, due to the resistance of the air to the lowering of the particle;

On the basis of the Newton's second law and the forces acting on the particle $A$, the equations of its motion in projections on coordinate axes were made. The driving aerodynamic force $F_{A}$ occurs due to the movement of a point $A$ in the air flow. The driving force $F_{A}$ is decomposed into normal and tangential components [2]. 


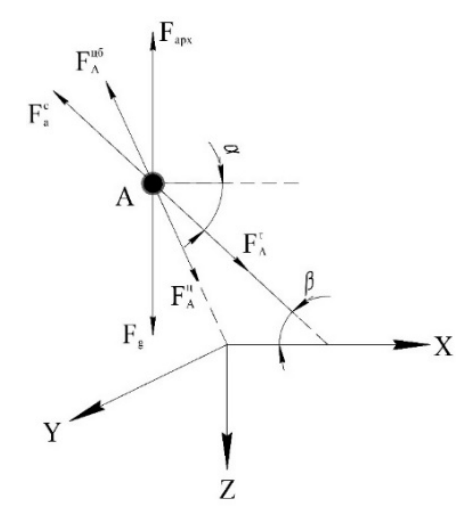

$\alpha$-angle between the direction of the absolute speed of the point $A$ and the plane $O X Z$;

$\beta$-angle between the direction of the absolute speed of the point $A$ and the plane $O X Z$;

$\bar{F}_{A}^{u} I I \bar{F}_{A}^{u \sigma} ; \bar{F}_{g} I I \bar{F}_{a p x} I I O Z$; forces $\bar{F}_{a}^{c} I I \bar{F}_{A}^{\tau}$ are directed under the angles $\alpha$ to $O X$ and $\beta$ to $O Y$

Fig. 2. Forces acting to the particle $A$

To analyze the trajectories of seed movement, we placed them at the junction of the inlet pipe and in the chambers of the centrifugal distributor (figure 3). To simplify the mathematical model, we assume that seeds from all five points are immediately involved in rotational motion along the walls of the distributor chamber, descending to the discharge pipes [3].
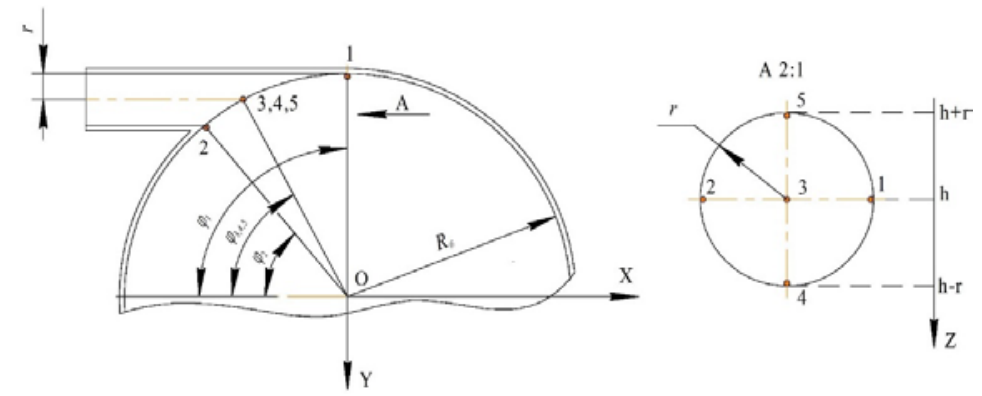

$\varphi_{1}, \varphi_{2}, \varphi_{3,4,5}$ - angular coordinate of the seeds relative to the axes $X Y ; h$ - height of the distributor on the axis $Z, r$-radius of the inlet pipe; $R_{\sigma}$ - radius of the distributor.

Fig. 3. Coordinates of the analyzed seeds

Let's analyze the forces acting on the seeds in the centrifugal distributor [4]. The tangential component of the driving aerodynamic force is defined by the expression:

$$
F_{A}^{\tau}=C_{\mathcal{C}} S_{c} \rho_{s} \frac{u_{b}^{2}}{2}
$$

where $C_{c}$ - coefficient of the aerodynamic drag of the particle;

$\rho_{\theta}$ - air density, $\mathrm{kg} / \mathrm{m}^{3}$;

$u_{s}$ - air velocity, $\mathrm{m} / \mathrm{s}$;

$S_{c}$ - area of midpoint section of the particle, $\mathrm{m}^{2}$

Area of midpoint section of the particle is expressed as: 


$$
S_{c}=\frac{3 m_{c}}{4 R_{c} \rho_{c}}
$$

where $m_{c}$ - weight of a single seed, $\mathrm{kg}$;

$R_{c}$ - radius of a single seed, $\mathrm{m}$;

$\rho_{c}$ - seed density, $\mathrm{kg} / \mathrm{m}^{3}$;

With 2.7 in mind, the expression 2.6 will take the form:

$$
F_{A}^{\tau}=\frac{3 m_{c} C_{c} u_{b}^{2} \rho_{b}}{8 R_{c} \rho_{c}}
$$

Centrifugal component of the driving aerodynamic force:

$$
F_{A}^{u \sigma}=m_{c} \alpha^{2} R_{\sigma}^{2}
$$

where $\alpha$ - angle between the speed direction of point A when entering the distributor; $R_{\sigma}$ - radius of the seed distributor

The strength of the aerodynamic grain drag:

$$
F_{a}^{c}=C_{c} S_{c} \frac{\rho_{B}}{2}\left(u_{s}-u_{c}\right) \sqrt{\left(u_{z}^{B}-u_{z}^{c}\right)^{2}+\left(u_{y}^{b}-u_{y}^{c}\right)^{2}+\left(u_{x}^{b}-u_{x}^{c}\right)^{2}}
$$

where $u_{z}^{6}, u_{y}^{6}, u_{x}^{B}$ - components of the air flow velocity;

$u_{z}^{c}, u_{y}^{c}, u_{x}^{c}$ - components of the grain velocity.

The centrifugal force of Archimedes:

$$
F_{A}^{u}=-\frac{\rho_{\theta} u_{\theta}^{2} m_{c}}{\rho_{c} R_{\sigma}}
$$

Gravity:

$$
F_{g}=m_{c} g
$$

The lifting force of Archimedes:

$$
F_{a p x}=-\frac{m_{c} \rho_{\theta} g}{\rho_{c}}
$$

where $u_{\theta}$ - air velocity, $\mathrm{m} / \mathrm{s}$;

$u_{c}$ - seed velocity, $\mathrm{m} / \mathrm{s}$;

$u$ - average air velocity, $\mathrm{m} / \mathrm{s}$;

$g$-acceleration of free falling $\mathrm{m} / \mathrm{s}^{2}$;

Force projections on the coordinate axis: 


$$
\left\{\begin{array}{l}
m a_{x}=F_{A}^{\tau} \cos \beta \cos \left(\alpha_{0}+\omega t\right)-F_{A}^{u \sigma} \cos \beta \cos \left(\varphi_{0}+\omega t\right)-F_{a}^{c} \cos \beta \cos \left(\alpha_{0}+\omega t\right)+ \\
+F_{A}^{u} \cos \beta \cos \left(\varphi_{0}+\omega t\right) \\
m a_{y}=F_{A}^{\tau} \cos \beta \sin \left(\alpha_{0}+\omega t\right)-F_{A}^{u \sigma} \cos \beta \sin \left(\varphi_{0}+\omega t\right)-F_{a}^{c} \cos \beta \sin \left(\alpha_{0}+\omega t\right)- \\
-F_{A}^{u} \sin \left(\varphi_{0}+\omega t\right) \\
m a_{z}=F_{g}+F_{A}^{\tau} \sin \beta \cos \left(\alpha_{0}+\omega t\right)-F_{a p x}-F_{A}^{u \sigma} \sin \beta \sin \left(\varphi_{0}+\omega t\right)
\end{array}\right.
$$

The forces $F_{a}^{c}, F_{A}^{u}, F_{a p x}$ have a negligible effect on the particle $A$ and will not be taken into account in the further descriptions of the motion process, with this in mind, we will write a system of equations:

$$
\left\{\begin{array}{l}
m a_{x}=F_{A}^{\tau} \cos \beta \cos \left(\alpha_{0}+\omega t\right)-F_{A}^{u \sigma} \cos \beta \cos \left(\varphi_{0}+\omega t\right) \\
m a_{y}=F_{A}^{\tau} \cos \beta \sin \left(\alpha_{0}+\omega t\right)-F_{A}^{u \sigma} \cos \beta \sin \left(\varphi_{0}+\omega t\right) \\
m a_{z}=F_{g}+F_{A}^{\tau} \sin \beta \cos \left(\alpha_{0}+\omega t\right)-F_{A}^{u \sigma} \sin \beta \sin \left(\varphi_{0}+\omega t\right)
\end{array}\right.
$$

Substituting formulas 4, 5, 8 in the system of equations we get:

$$
\left\{\begin{array}{l}
m_{c} \frac{d V_{x}}{d t}=\frac{3 m_{c} C_{c} u_{s}^{2} \rho_{B}}{8 R_{c} \rho_{c}} \cos \beta \cos \left(\alpha_{0}+\omega t\right)-m_{c} \omega^{2} R_{\sigma} \cos \beta \cos \left(\varphi_{0}+\omega t\right) \\
m_{c} \frac{d V_{y}}{d t}=\frac{3 m_{c} C_{c} u_{b}^{2} \rho_{b}}{8 R_{c} \rho_{c}} \cos \beta \sin \left(\alpha_{0}+\omega t\right)-m_{c} \omega^{2} R_{\sigma} \cos \beta \sin \left(\varphi_{0}+\omega t\right) \\
m_{c} \frac{d V_{z}}{d t}=m_{c} g+\frac{3 m_{c} C_{c} u_{b}^{2} \rho_{b}}{8 R_{c} \rho_{c}} \sin \beta \cos \left(\alpha_{0}+\omega t\right)-m_{c} \omega^{2} R_{\sigma} \sin \beta \sin \left(\varphi_{0}+\omega t\right)
\end{array}\right.
$$

We exclude the mass of the seed $m_{c}$ from the equations and denote

$$
\begin{gathered}
A=\frac{3 C_{c} u_{b}^{2} \rho_{b}}{8 R_{c} \rho_{c}}, B=\omega^{2} R_{\sigma}: \\
\left\{\begin{array}{l}
\frac{d V_{x}}{d t}=A \cos \beta \cos \left(\alpha_{0}+\omega t\right)-B \cos \beta \cos \left(\varphi_{0}+\omega t\right) \\
\frac{d V_{y}}{d t}=A \cos \beta \sin \left(\alpha_{0}+\omega t\right)-B \cos \beta \sin \left(\varphi_{0}+\omega t\right) \\
\frac{d V_{z}}{d t}=m_{c} g+A \sin \beta \cos \left(\alpha_{0}+\omega t\right)-B \sin \beta \sin \left(\varphi_{0}+\omega t\right)
\end{array}\right.
\end{gathered}
$$

We integrate expression 13 and get the particle velocity in the centrifugal seed distributor [2]:

$$
\left\{\begin{array}{l}
\int \frac{d V_{x}}{d t}=A \cos \beta \cos \left(\alpha_{0}+\omega t\right) d t-B \cos \beta \cos \left(\varphi_{0}+\omega t\right) d t \\
\int \frac{d V_{y}}{d t}=A \cos \beta \sin \left(\alpha_{0}+\omega t\right) d t-B \cos \beta \sin \left(\varphi_{0}+\omega t\right) d t \\
\int \frac{d V_{z}}{d t}=m_{c} g d t+A \sin \beta \cos \left(\alpha_{0}+\omega t\right) d t-B \sin \beta \sin \left(\varphi_{0}+\omega t\right) d t
\end{array}\right.
$$


Having integrated 14 by time $t$ we receive:

$$
\left\{\begin{array}{l}
V_{x}=\frac{A \cos \beta \sin \left(\alpha_{0}+\omega t\right)}{\omega}-\frac{B \cos \beta \sin \left(\varphi_{0}+\omega t\right)}{\omega}+C_{1}^{x} \\
V_{y}=-\frac{A \cos \beta \cos \left(\alpha_{0}+\omega t\right)}{\omega}+\frac{B \cos \beta \cos \left(\varphi_{0}+\omega t\right)}{\omega}+C_{1}^{y} \\
V_{z}=g t+\frac{A \sin \beta \sin \left(\alpha_{0}+\omega t\right)}{\omega}+\frac{B \sin \beta \cos \left(\varphi_{0}+\omega t\right)}{\omega}+C_{1}^{z}
\end{array}\right.
$$

To determine the integration constants in the particle $A$ velocity equations, we substitute the value 15 of the initial conditions [2,8]: at $t=0: V_{x 0}=u$

$$
\left\{\begin{array}{l}
C_{1}^{x}=u-\frac{A \cos \beta \sin \left(\alpha_{0}\right)}{\omega}+\frac{B \cos \beta \sin \left(\varphi_{0}\right)}{\omega} \\
C_{1}^{y}=\frac{A \cos \beta \cos \alpha_{0}}{\omega}-\frac{B \cos \beta \cos \varphi_{0}}{\omega} \\
C_{1}^{z}=-\frac{A \sin \beta \sin \left(\alpha_{0}\right)}{\omega}-\frac{B \sin \beta \cos \left(\varphi_{0}+\omega t\right)}{\omega}
\end{array}\right.
$$

To determine the trajectory of the particle $A$, we integrate the system of equations 15 in time $t$ :

$$
\left\{\begin{array}{l}
x=-\frac{A \cos \beta \cos \left(\alpha_{0}+\omega t\right)}{\omega^{2}}+\frac{B \cos \beta \cos \left(\varphi_{0}+\omega t\right)}{\omega^{2}}+C_{1}^{x} t+C_{2}^{x} \\
y=-\frac{A \cos \beta \sin \left(\alpha_{0}+\omega t\right)}{\omega^{2}}+\frac{B \cos \beta \sin \left(\varphi_{0}+\omega t\right)}{\omega^{2}}+C_{1}^{y} t+C_{2}^{y} \\
z=\frac{g t^{2}}{2}-\frac{A \sin \beta \cos \left(\alpha_{0}+\omega t\right)}{\omega^{2}}+\frac{B \sin \beta \sin \left(\varphi_{0}+\omega t\right)}{\omega^{2}}+C_{1}^{z} t+C_{2}^{z}
\end{array}\right.
$$

To determine the integration constants in the equations of the particle $A$ trajectory we substitute 17 in the values of the initial conditions: $t=017, x_{0}=0, z_{0}=0, y_{0}=-R_{\sigma}$ :

$$
\left\{\begin{array}{l}
C_{2}^{x}=\frac{A \cos \beta \cos \left(\alpha_{0}\right)}{\omega^{2}}-\frac{B \cos \beta \cos \left(\varphi_{0}\right)}{\omega^{2}} \\
C_{2}^{y}=-R_{6}+\frac{A \cos \beta \sin \alpha_{0}}{\omega^{2}}-\frac{\left.B \cos \beta \sin \varphi_{0}\right)}{\omega^{2}} \\
C_{2}^{z}=\frac{A \sin \beta \cos \left(\alpha_{0}\right)}{\omega^{2}}-\frac{B \sin \beta \sin \left(\varphi_{0}+\omega t\right)}{\omega^{2}}=0
\end{array}\right.
$$

The values of integration constants calculated from expressions 16 and 18 are shown in table 1 .

Table 1. Constants of integration

\begin{tabular}{|l|c|c|c|c|c|c|}
\hline \multicolumn{1}{|c|}{ Constant of integration } & $C_{1}^{x}$ & $C_{1}^{y}$ & $C_{1}^{z}$ & $C_{2}^{x}$ & $C_{2}^{y}$ & $C_{2}^{z}$ \\
\hline Value & 0 & 0 & 0 & 0 & 0 & 0 \\
\hline
\end{tabular}

In the system of equations 17 describing the seed movement in the centrifugal seed distributor, the values of angles and coefficients were substituted [5]. 
To analyze the nature of the particle $A$ movement in the centrifugal seed distributor, graphs of changes in the particle coordinates from time (figure 4) were constructed for the specified parameters given in table 2 .

Table 2. Parameters of the centrifugal distributor

\begin{tabular}{|l|c|}
\hline Parameter & Value \\
\hline Radius of the distributor $R_{\sigma}, \mathrm{m}$ & 0,2 \\
\hline Radius of inlet pipe $r, \mathrm{~m}$ & 0,04 \\
\hline Air density $\rho_{\sigma}$ & 0,22 \\
\hline Specific seed density $\rho_{c}$ & 800 \\
\hline $\begin{array}{l}\text { Initial angle between the direction of absolute velocity of } \\
\text { the point } A \text { and plane } O X Z \beta_{0}, \text { radian }\end{array}$ & 0,122 \\
\hline $\begin{array}{l}\text { Angle between the direction of absolute velocity of the } \\
\text { point } A \text { and plane } O X Z ; \beta, \text { radian }\end{array}$ & 1,571 \\
\hline Angular coordinate of the point $1 \varphi_{1}$, radian & 0,6434 \\
\hline Angular coordinate of the point $2 \varphi_{2}$, radian & 0,927 \\
\hline Angular coordinate of the points 3,4 and $5 \varphi_{3,4,5}$, radian & 0.8 \\
\hline Height of the distributor $h, \mathrm{~m}$ & 15 \\
\hline Velocity of seed movement $u, \mathrm{~m} / \mathrm{s}$ & 0 \\
\hline $\begin{array}{l}\text { Initial angle between the direction of absolute velocity of } \\
\text { the seed } A \text { and the } \alpha_{0} \text { plane; the plane } O X Z \text {, radian }\end{array}$ & 0,0019 \\
\hline Equivalent radius of seeds $r_{c}, \mathrm{~m}$ & 0,21 \\
\hline Coefficient of aerodynamic drag of a particle $C_{c}$ & \\
\hline
\end{tabular}

Using the specified values in the MathCad program, graphs of changes in the coordinates of point $A$ by time $t$ were constructed [6,7].

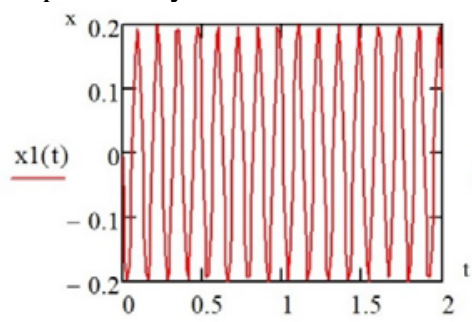

a)

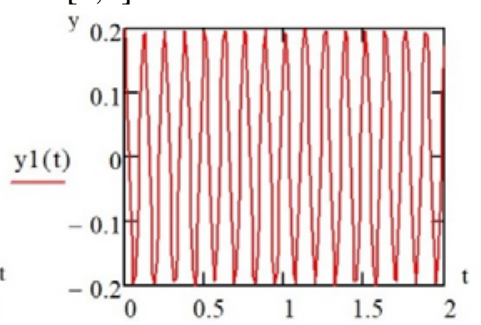

6)

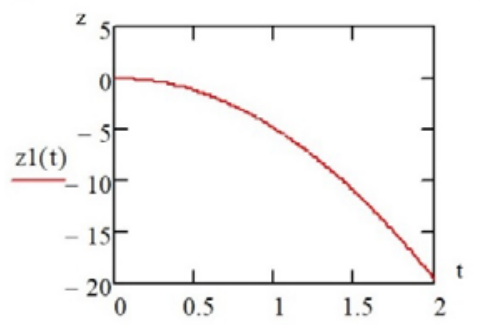

B)

a - change of coordinates in the axis $X$; $\sigma$ - change of coordinates in the axis $Y$; change of coordinates in the axis $Z$

Fig. 4. Coordinates of the particle from $A$ in time $t$ 
The coordinates of the point along the axes $X$ and $Y$ over time (figure $4 \mathrm{a}$ and b) are changed in a range equal to the radius $R_{\sigma}$ of the seed distributor [8], and the point descends along the axis $Z$ (figure $4 \mathrm{c}$ ).

Graphs of changes in the coordinates of points 2,3,4,5 are identical and are shown in Appendix A. In the MathCad program [9], seed trajectories were constructed in the centrifugal seed distributor (figure 5). The seed trajectories are spirals that are swirled around the $Z$-axis $[10,11]$.

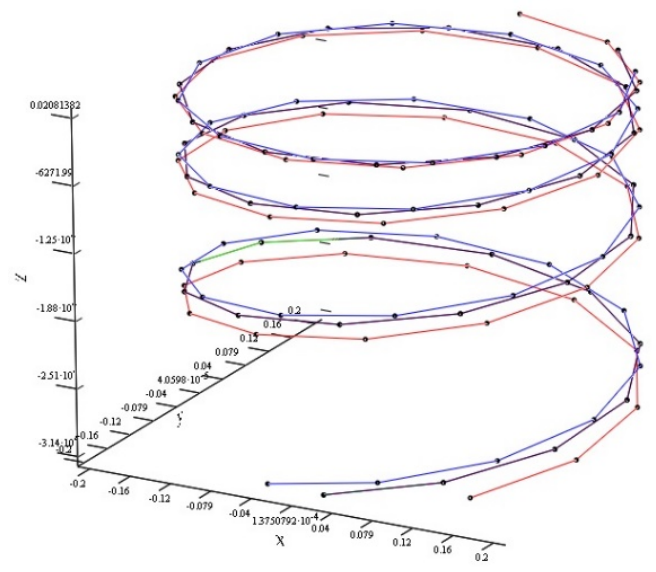

Fig. 5. Trajectory of seeds in a centrifugal seed distributor

According to figure 5, the seeds move around the $Z$ axis within the radius of the distributor $R_{\sigma}$ and fall down the height of the distributor $h$, taking this into account, we rewrite the system of equations 17, equating the coordinates $x=R_{\sigma}, y=R_{\sigma}$ and $z=h$ :

$$
\left\{\begin{array}{l}
R_{\sigma}=-\frac{A \cos \beta \cos \left(\alpha_{0}+\omega t\right)}{\omega^{2}}+\frac{B \cos \beta \cos \left(\varphi_{0}+\omega t\right)}{\omega^{2}} \\
R_{\sigma}=-\frac{A \cos \beta \sin \left(\alpha_{0}+\omega t\right)}{\omega^{2}}+\frac{B \cos \beta \sin \left(\varphi_{0}+\omega t\right)}{\omega^{2}} \\
h=\frac{g t^{2}}{2}-\frac{A \sin \beta \cos \left(\alpha_{0}+\omega t\right)}{\omega^{2}}+\frac{B \sin \beta \sin \left(\varphi_{0}+\omega t\right)}{\omega^{2}}
\end{array}\right.
$$

The formula expressed from the first equation of the system 19:

$$
\cos \left(\alpha_{0}+\omega t\right)=\frac{B \cos \left(\varphi_{0}+\omega t\right)}{A}-\frac{R_{\tilde{\sigma}} \omega^{2}}{A \cos \beta}
$$

The formula 20 was substituted into the third equation of the system 19 and the equation was obtained:

$$
h=\frac{g t^{2}}{2}-\frac{A \sin \beta\left\{\frac{B \cos \left(\varphi_{0}+C t\right)}{A}-\frac{R_{\delta} \omega^{2}}{A \cos \beta}\right\}}{\omega^{2}}+\frac{B \sin \beta \sin \left(\varphi_{0}+\omega t\right)}{\omega^{2}}
$$

Substitute the values $A$ and $B$ in equation 21 to get: 


$$
h=\frac{g t^{2}}{2}-\frac{\frac{3 C_{c} u_{s}^{2} \rho_{b}}{8 R_{c} \rho_{c}} \sin \beta\left\{\frac{8 R_{c} \rho_{c} \omega^{2} R_{b} \cos \left(\varphi_{0}+\omega t\right)}{3 C_{c} u_{b}^{2} \rho_{b}}-\frac{8 R_{c} \rho_{c} R_{b} \omega^{2}}{3 C_{c} u_{b}^{2} \rho_{b} \cos \beta}\right\}}{\omega^{2}}+\frac{\omega^{2} R_{b} \sin \beta \sin \left(\varphi_{0}+\omega t\right)}{\omega^{2}}
$$

The resulting equation describes the relationship between the geometric and operating parameters of the centrifugal seed distributor, it is nonlinear, and is solved using a computer.

\section{Conclusions}

The proposed design and technological scheme of the seed distributor of a pneumatic grain seeder will increase the uniformity of the seed distribution along the seed pipes. Theoretical studies of the centrifugal seed distributor allowed us to obtain equations describing the process of seed movement in the centrifugal seed distributor of a pneumatic grain seeder. The relationship between the operating and design parameters of the centrifugal distributor is determined. A mathematical model of the technological process of operation of the centrifugal distributor of the grain pneumatic seeder of central dosing is developed, taking into account the design and operating parameters.

\section{References}

1. Bosoy E.S. Theory, construction and calculation of agricultural machines [Text] / E. S. Bosoy, I. I. Smirnov, O. V. Vernyaev, E. G. Sultan-Shah. - Moscow: "Engineering", $1978-568$ p.

2. Vygodsky M. Ya. Handbook of higher mathematics [Text] / M. Ya. Vygodsky. - M.: AST: Astrel, 2006. - $991 \mathrm{p}$.

3. Maslov G. G. Innovative technology in energy-saving technologies of field cultivation [Text] / G. G. Maslov, A. S. Serguntsov // Proceedings of Kuban State Agrarian University. 2018. \# 71. P. 110-117.

4. Pat. 2448444 Russian Federation, IPC A01S15 / 04. Pneumatic seeder with a central dosing system [Text] / E. I. Trubilin, A.V. Khokhlov, A. A. Khokhlov, A. E. Bogus, V. V. Kutseev; applicant and patent holder Federal State Budgetary Educational Institution of higher education "Kuban State Agrarian University named after I. T. Trubilin". \# 2010133832/13; declared 12.08.2010; publ. 27.04.2012, bul. \#. 12. - 13 p.: ill.

5. Rybalkin P. N. New adaptive energy-and soil-saving technologies of winter wheat and corn cultivation in Krasnodar Territory [Monograph] / P. N. Rybalkin P. N. [et al]. Krasnodar: Russian Academy of Agricultural Sciences, Krasnodar Scientific Research Institute of Agriculture named after P. P. Lukyanenko, LLC "Prosveschenie-Yug", 2002, 103 p.

6. Rybalkin P. N. Agrotechnical requirements for basic technological operations in adaptive technologies of winter wheat and corn cultivation and new technical units for their implementation in Krasnodar Territory [Recommendations] / P. N. Rybalkin P. N. [et al]. - Krasnodar: Department of Agriculture and Food of Krasnodar Territory, Russian Academy of Agricultural Sciences, Krasnodar Research Institute of Agriculture named after P. P. Lukyanenko., Ph LLC "Agroprompoligrafist", 2001. - 144 p.

7. Agricultural machines (device, operation and basic adjustments): manual / V. A. Romanenko [et al]. - Krasnodar: KubSAU, 2014. - 232 p.

8. Sokht K. A. Statistical methods of researches of processes and machines in agribusiness [Text] / K. A. Sokht, E. I. Trubilin, V. I. Konovalov / / - Krasnodar: KubSAU, 2016. 217 p. 
9. Belousov S.V. Explanation of the angle of sharpening of a plough cutting working body Belousov S.V., Saprykin E.A., Karmazin I.S. B сборнике: E3S Web of Conferences 2019. C. 00025.

10. Belousov S.V. Design of the universal agricultural working body and study of its parameters Belousov S.V., Pomelyayko S.A., Novikov V.V. В сборнике: MATEC Web of Conferences 2018. C. 05006.

11. Belousov S.V. Theoretical justification of fruit separation process by a planetary fruit separator Belousov S.V., Gusak E.S. В сборнике: E3S Web of Conferences 2019. C. 00056. 